\title{
CRITICAL-POINT BEHAVIOUR IN SELF-ORGANIZING SYSTEMS: THE SEPTEMBER 1999 ATHENS EARTHQUAKE AND STOCK MARKET CORRECTION
}

\author{
Tzanis A. ${ }^{1}$ \\ ${ }^{1}$ Department of Geophysics - Geothermy,University of Athens, atzanis@geol.uoa.gr
}

\begin{abstract}
A quorum of observational and theoretical evidence indicates that seismogenesis is a critical phenomenon culminating with a large event that corresponds to some critical point: large earthquakes are preceded by the self-organization of the fault network and power-law acceleration of the seismic release rates. The stock market is not very different: the (inter)action of the traders is usually irreversible and determines the value of the stock, which determines the actions of other traders etc. Thus, the stock market becomes a self-organizing system and exhibits strong analogies with other self-organizing systems, such as earthquakes. Positive feed-back is also important and may lead to trend-chasing and rapid escalation of stock price. There is ample evidence that market crashes and large corrections are often preceded by speculative bubbles with power law acceleration of the market price. In diabolic coincidence, both such crises were observed in Athens during the September of 1999. On 7/9 a moderate (M5.9) but destructive earthquake; around 22/9 the Athens Stock Exchange rally of the past few months crashed. Both phenomena were preceded by characteristic power-law acceleration, respectively in crustal deformation and market growth. Two very different complex dynamic systems are shown to exhibit similar collective behaviour, pointing out the apparent universality of natural order.
\end{abstract}

Key words: Complex systems, Earthquake prediction, Econophysics, Stock Market Bubbles.

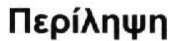

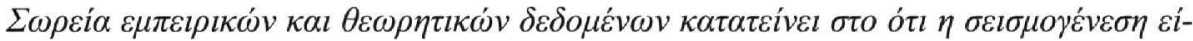

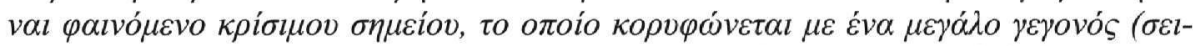

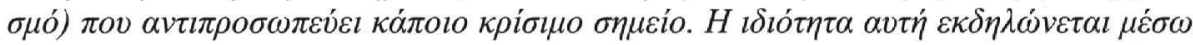

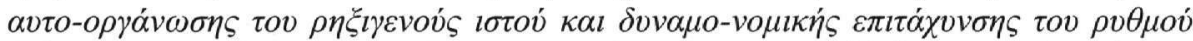

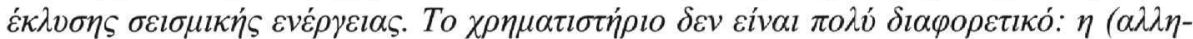

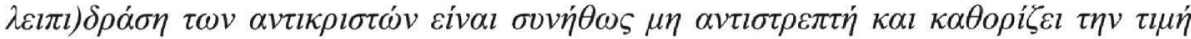

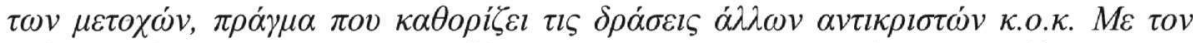

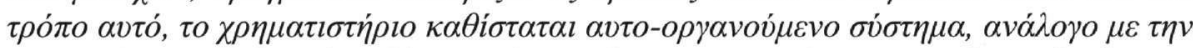

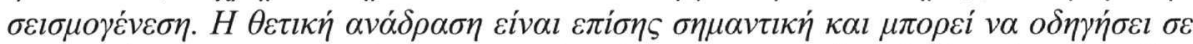

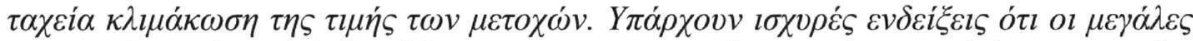

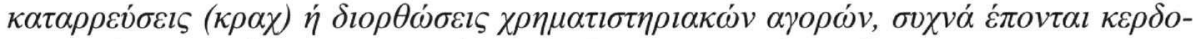

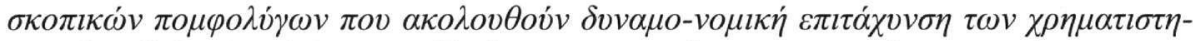

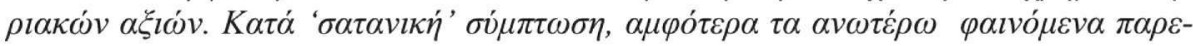




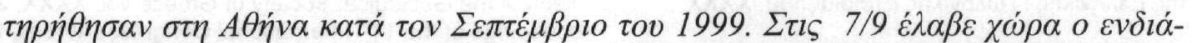

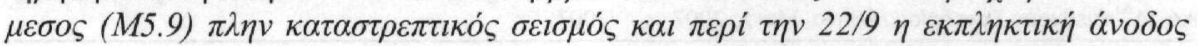

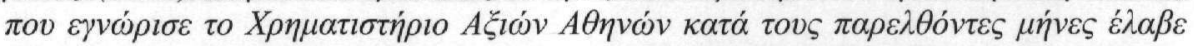

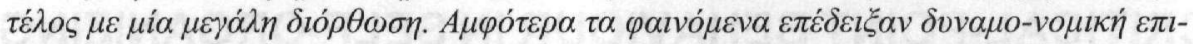

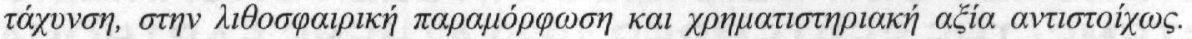

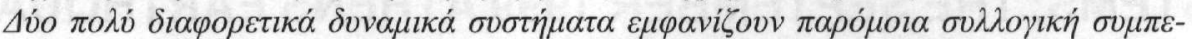

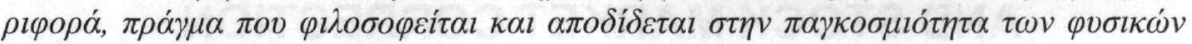

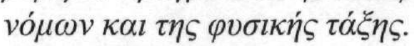

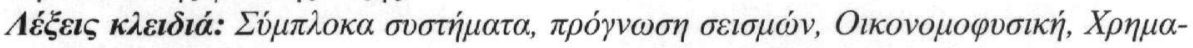

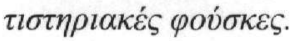

\section{Introduction}

It has been credibly argued that rupture in heterogeneous media is a critical phenomenon (e.g. Herrmann and Roux 1990, Vanneste and Sornette 1992, Sornette et al. 1992), and that the earthquake generation process can also be viewed as a critical phenomenon culminating with a large event that corresponds to some critical point (Keilis-Borok 1990, Sornette and Sammis 1995, Saleur et al. 1996a, 1996b, Bowman et al. 1998, Rundle et al. 2000 and many others). According to the Critical Point (CP) earthquake hypothesis, failure in the crust can be thought of as a scaling up process in which failure at one scale of a fault network is part of the damage accumulation over a larger scale, leading to long range stress-stress correlation and power-law increase (acceleration) of seismic release rates prior to a large earthquakes. The culminating large event will only occur when the network is in a critical state, balancing at the verge of disorder and characterised by both extreme susceptibility to external factors and strong correlation between its different parts.

As Sammis and Sornette (2001) point out, the CP model is fundamentally different from the principle of Self-Organised Criticality, according to which earthquakes evolve spontaneously in a statistically stationary critical state of the Earth's crust. The SOC doctrine holds that all events belong to the same global population and participate in shaping the self-organized critical state. In this view earthquakes are inherently unpredictable, because any small spontaneous instability has a chance of cascading into a large event. However, SOC processes are Abelian, while seismogenesis is characteristically non-Abelian. Conversely, in the (non-Abelian) CP view, a great earthquake represents the end of a cycle on its associated fault network and the beginning of a new one. The dynamic organization of the crust is not statistically stationary, but evolves as the cycle progresses and a great earthquake becomes more probable, thereby rendering possible the prediction of the cycle's end by monitoring the approach of the fault network toward a critical state.

The stock market is a totally different realm but recent research has shown that it exhibits remarkable analogy to well-known models in statistical physics and that it may also behave like a CP system (e.g. Feigenbaum and Freund 1996, Vandewalle et al. 1998, Johansen and Sornette 1998, 1999, 2000 and others). The analogy was based on a large amount of work exploiting the similarities between statistical physics and financial markets (e.g. Anderson et al. 1998, Kertesz and Kondor 1998). As Johansen and Sornette (1999) point out, at a 'microscopic' level, the individual trader can only have 3 possible states (sell, buy or stay neutral) and the transition from one state to another requires another trader and is usually irreversible (non-Abelian). Individual traders only have information on the action of a limited number of other traders and in general, only see the cooperative response of the market. The actions of the traders determine the value of the stock, which determines the actions of other traders etc. Thus, the stock market becomes a selforganizing system and it is natural to think of it in terms of other self-organizing systems, such as earthquakes, avalanches, crack propagation, etc. Positive feed-back is also important and present. Under favourable conditions, a strong prospect of profit may enhance positive feedback and enforce a strong upward trend in stock price, (trend-chasing), which will continue for as long as investors and traders can sustain market growth with fresh capital. However, if a given threshold is exceeded, a crash may follow. A series of recent publications have presented evidence that market 
crashes and large corrections are often preceded by speculative bubbles with power law acceleration of the market price.

In diabolic coincidence, both such crises occurred in Athens, Greece, during the fateful September of 1999. On 7 September Athens was rocked by a moderate albeit destructive earthquake. Around 22 September, the Athens Stock Exchange (ASE) price rally of the past few months ended with a large correction, followed by a period of instability and eventual collapse. Both phenomena were preceded by characteristic power-law acceleration, respectively in crustal deformation (Benioff strain) and market growth as indicated by the composite ASE General Index. As easily shown, in both cases the culmination (earthquake, correction) was foreseeable in advance of the corresponding crises.

Two very different natural many-component systems, one wholly inanimate and one comprising of living and symbolically reasoning beings, exhibit a similar collective behaviour. This paper discusses the underlying processes and presents an analysis of the events leading to the two crises. Moreover, notwithstanding the particular characteristics of either system, it is worth pointing out the apparent universality of natural order. This type of philosophical ramification will also be considered.

\section{The Earthquake}

\subsection{Generalities}

The power-law acceleration of seismic release rates prior to a large earthquake may be described by the time-to-failure relation of the form

$\sum \Omega(t)=K+A \cdot\left(t_{c}-t\right)^{n}$

where $\Omega$ is any quantity estimated from the earthquake magnitude using an expression of the form

$\log _{10} \Omega=c \cdot M+d$.

$\Sigma \Omega(t)$ is the cumulative seismic release, $t_{c}$ is the critical time at which a critical state is attained, $K=\sum \Omega\left(t=t_{c}\right), A$ is negative and $n<1$. This scaling law has been justified in terms of run-away crack propagation and empirical expressions for accelerating (tertiary) creep preceding failure in the laboratory (Voight 1989, Varnes 1989, Bufe and Varnes 1993), but it can also result naturally from the many-body interactions between small cracks forming before the impending rupture (Sornette and Sammis 1995, Saleur et al. 1996a, 1996b, Bowman et al. 1998). In the latter, small and intermediate size events are associated with the increasing correlation length of the regional stress, while the culminating earthquake in the cycle represents the critical point occurring when the system is correlated over long ranges.

Depending on the coefficients $c$ and $d$ in (2), the seismic release rates can be moment or energy ( $c=1.5$ ), Benioff strain, (square root of energy, $c=0.75)$, or event count $(c=0, d=1)$. Previous work has determined that the cumulative Benioff strain is particularly useful when smaller events are also of interest and magnitude scaling is desirable, while cumulative moment is dominated by the larger earthquakes and event count does not allow for magnitude scaling. In that case (1) reads

$\varepsilon(t)=\sum_{i=1}^{N(t)} \sqrt{E_{i}(t)}=K+A\left(t_{c}-t\right)^{n}$,

where $E_{i}(t)$ the energy of the $i^{\text {th }}$ event and $N(t)$ is the total number of events at time $t$. Earlier work has empirically determined that typically, $n \approx 0.3$. There are also two theoretical predictions for the value of the critical exponent. Using a model seismogenic crust governed a particular damage rheology, Ben-Zion and Lyakhovsky (2002) derive a non-singular power law relation for cumulative Benioff strain proportional to $\left(t_{c}-t\right)^{1 / 3}$, i.e. $n=1 / 3$. Rundle et al. (2000) adopt a very different approach by relating the behaviour of seismicity prior to a large earthquake to the excitation in proximity of a spinodal instability. They show that the power-law activation associated with the 
spinodal instability is essentially identical to the power-law acceleration of Benioff strain observed prior to earthquakes, and that $n=0.25$.

\subsection{Data analysis and results}

The seismicity data used in this study are extracted from the raw catalogue of the Geodynamics Institute of the National Observatory of Athens (NOA, http://www.gein.noa.gr/services/cat.html) and span the period $1 / 10 / 1998-16 / 10 / 1999$. The properties of this data set have been extensively investigated by Tzanis and Makropoulos (2002), who have shown that for the period in question the NOA catalogue is remarkably homogeneous and has a threshold of completeness $M_{L} \approx 2.8$. The energy $E_{i}(t)$ required by equation (3) can be estimated after Gutenberg and Richter (1956) as

$\log _{10} E_{i}(t)=4.8+1.5 \cdot M_{S}$,

which yields

$\log _{10} \varepsilon(t)=\log _{10} \sqrt{E_{i}(t)}=2.4+0.75 M_{S}$.

Kanamori and Anderson (1975) have shown that (4a) is consistent with what is expected theoretically for a classical crack model with a constant stress drop. The stress drop does not vary significantly with earthquake size Hanks 1977), at least down to seismic moments of the order $5 \times 10^{18}$ ergs, $\left(M_{W} \sim 3\right.$ or $M_{S} \sim 1.5$, e.g. Hanks 1977). Thus, equations (4) comprise energy - magnitude scaling laws applicable over a broad range of magnitudes. Since NOA reports local magnitudes, it is necessary to convert $M_{L}$ to $M_{S}$. Tzanis and Vallianatos (2003) have worked out the relationship $M_{S}=M_{L} \times 1.687 \pm 0.053-3.35 \pm 0.263$.

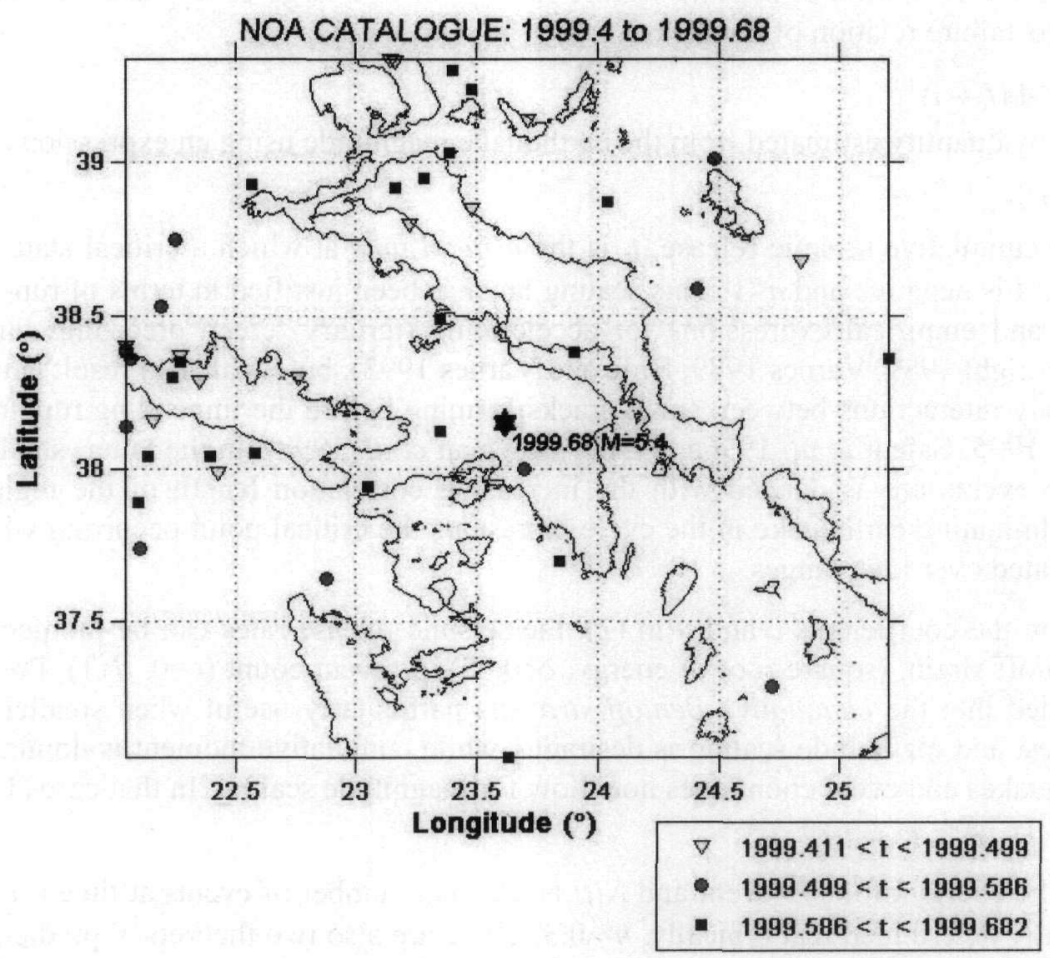

Figure 1 - Excerpt form the catalogue of the Geodynamics Institute, National Observatory of Athens spanning the interval 1999.4-1999.68. Earthquake epicentres within a radius of 140 km around the 7/9/99 M5.4 Athens earthquake are shown in chronological order. Magnitude scale is the $M_{L}$ scale reported by NOA 
The identification of power-law behaviour in seismic release rates is rather straightforward: the cumulative Benioff strain vs. time for earthquakes within a circle of radius $R$ is modelled with the time-to-failure power-law relation (3), using a Hedgehog non-linear optimisation procedure operating on the $\mathrm{L}_{2}$ norm. At the critical time $t_{c}$, equation (3) reduces to $\varepsilon\left(t=t_{c}\right)=K$. Therefore, at any $t \rightarrow t_{c}$, equation (4b) can be used to obtain a predicted magnitude as

$$
\hat{M}_{S}=\left(\log _{10}[K-\varepsilon(t)]-2.4\right) / 0.75 \text {. }
$$

Following Bowman et al. (1998), the performance of the power law fit against the null hypothesis of constant seismic release rate, is quantified by defining a curvature parameter

\section{$C=$ (Power law fit RMS) / (Linear fit RMS),}

such, that when the data are best described by a power-law curve, the RMS error will be small compared to the RMS error of the linear fit and $C$ will also be small. The radius $R_{c}$ such that $C\left(R_{c}\right)$ $=\min \{C(R)\}$ and the corresponding model parameters are deemed optimal: $R_{c}$ corresponds to our estimate of the correlation length of the self-organizing process, or critical radius.

Since this is a posterior analysis, the epicentre and $t_{c}$ are known. It is therefore reasonable to begin with constrained modelling in order to facilitate the determination of other important factors, such as are the correlation length $R_{c}$ and the predictable magnitude. Using the sub-catalogue illustrated in Figure 1 and pinning $t_{c}$ to 00:00 of 7/9/1999, one obtains $R_{c} \approx 110 \mathrm{~km}$ (for details see Tzanis and Makropoulos 2002).

With $R_{c}$ known, it is possible to drive a conditional running forecast of the main shock by calculating $C, t_{c}$, and $M_{S}$ as a function of the time-to-failure, in order to study the evolution of the self-organizing process. Two snapshots of this procedure are presented below. Figure 2 shows the state of affairs on 00:00 of 31 August 1999 and Figure 3 on 24:00 of 5 September 1999. As is apparent in Figure 2, a power-law acceleration of the seismic release rate if clearly developing around the main shock epicentre, although the predicted critical time and magnitude are not accurate enough ( -4.6 days with respect to the main shock and 5.65 respectively). In Figure 3, however, the acceleration process is fully developed and approaching the critical point; the predicted critical time and magnitude have improved significantly to -2.76 days with respect to the main shock and 5.81 respectively. No seismic activity is observed thereafter at the level of $M_{L}>2.8$, and the entire region within $100-120 \mathrm{~km}$ from the epicentre went quiescent almost 66 hours $(2.76$ days) prior to failure. This may imply that whatever stress-stress correlations and organization could be done, was actually completed by that time and the regional fault system had entered the critical phase, only waiting for some additional factor to instigate irreversible instability (rupture).

However, this is not the end of the story. One may observe in Figure 2, and more evidently in Figure 3, that the curvature of the acceleration process is very low up to the 17/8/99 M7.4 Izmit earthquake - indicating very low seismic release rate changes - and develops rigorously thereafter. This points to a causal relationship between the two earthquakes. Tzanis and Makropoulos (2002) have performed an extensive investigation of this possibility and have indeed shown that that prior to 17/8/99, power-law acceleration around Athens was subtle and could not be confidently detected. Precipitous power-law behaviour began immediately after the Izmit earthquake and culminated with the Athens event, disappearing soon afterward. This effect could clearly be observed over the entire North Aegean, the Sporadhes, Euboea, Attica and through the Central and SW Peloponnesus to the Ionian islands. It comprises ample evidence of a causal relationship between the two events, also involving stress-stress correlation and fault interaction over a broad area. Note also that evidence of dynamically triggered regional seismicity following the Izmit earthquake was independently presented by Brodsky et al. (2000).

According to the rate-and-state model of seismoegenesis, during the long interval of self-driven accelerating slip, a fault's sliding speed depends on the initial stress and state (fault conditions). After an external stress step is applied due to unstable sliding in another fault, the slip speed depends on the new stress and state. An earthquake (frictional instability) nucleates when the fault 
strength decreases more rapidly with slip than the elastic stiffness of the surrounding rocks (Gomberg et al. 1998). Thus, a stress increase, (positive step) will advance the time of failure by an amount depending on its magnitude and fault state, triggering an earthquake if the conditions are right. According to Gomberg et al. (1997), near (tens of kilometres) small/ moderate earthquakes and remote (thousands of kilometres) earthquakes with magnitudes 2 to 3 units larger may be equally effective at triggering seismicity. Notably, the rate-and-state model is the only able to account for remote interactions (e.g. Harris, 1998) and also predicts that the duration of the increased seismicity rates cannot exceed that of the transient load, consistently with the finite duration of accelerated seismicity observed between the Izmit and Athens events.

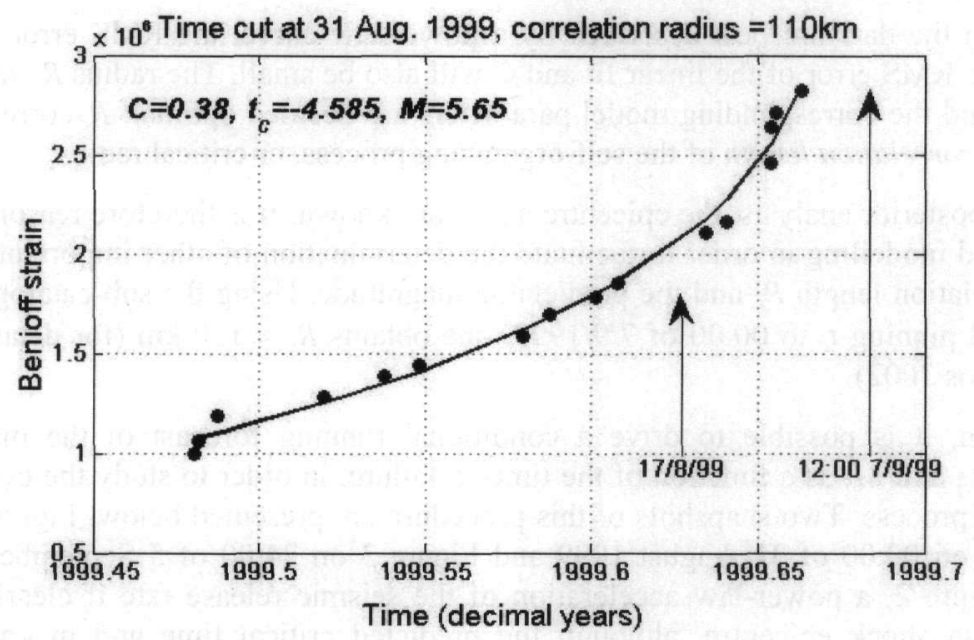

Figure 2 - The self-organizing process of the Athens earthquake at 00:00 of 31/8/1999. Earthquakes with $M_{L}>\mathbf{2 . 8}$ within a radius $R_{c}=110 \mathrm{~km}$ around the epicentre are used. The left arrow points to the origin time of the 17/8/1999 M7.4 Izmit, Turkey, event on the N. Anatolian fault, approx. $750 \mathrm{~km}$ to the NE. The right arrow points to the origin time of the Athens event

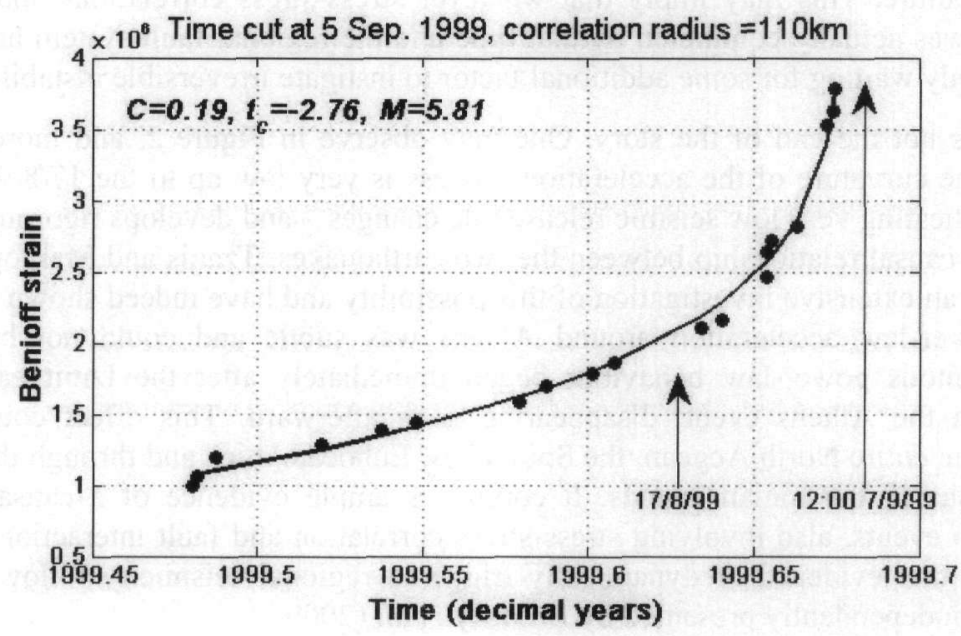

Figure 3 - The self-organizing process of the Athens earthquake at 24:00 of 5/9/1999. Earthquakes with $M_{L}>2.8$ within a radius $R_{c}=110 \mathrm{~km}$ around the epicentre are used. Annotations are as per Figure 2 above 
Note though, that while dynamic rate-and-state friction may provide a viable explanation of the observed acceleration of seismicity rates and the triggering of the Athens event, it cannot account for its power-law form over such a broad area. The answer may lie in the self-organisation of the regional fault system. A fundamental property of critical systems is their finite hierarchy. As Huang et al. (1998) ascertain, any given level of the hierarchical rupture is equivalent to a critical point for the lower levels. In a CP system without a single largest fault element to rupture, the long range correlation of the stress field may either allow the final event to connect neighbouring faults (as for instance in the case of the 1992 earthquake of Landers, California), or, may simply remove the system from criticality with a number of small(er) earthquakes. Thus, the transient stress load of the Izmit event may have perturbed the regional fault system, by triggering multiple distributed failures through the North Aegean and Continental Greece (we assume that very many more small faults were destabilised, than the number corresponding to the $M_{L}>2.8$ detectable by the NOA network). The ensuing period of accelerated seismicity can effectively be understood as a reorganisation process, whereby the stress redistribution produced by these failures triggered more faults, until the system reached a new state (small events smooth the stress field at long length scales by redistributing stress to neighbouring regions and are the agents by which long stress correlations are established). The many-fault interactions may account for the power-law behaviour of the accelerating seismic release. At Athens, premature failure may have been triggered in a fault pre-stressed to near its load bearing capacity. Supposing that this was the highest level of the perturbed system capable of rupturing at this given time, it may have served as the focal point for the self-(re)organisation process.

\section{The Stock Market}

\subsection{Generalities}

In earthquakes correlation arises from stress-stress interactions between faults (system elements) in a spatially extended system (the lithosphere), subject to continuous supply of stress (energy). The stock market differs in several ways. It functions within a more complex environment: human society. It involves investors and traders as elements and "space", "distance" and "scale" are immaterial measures determined by human interactions.

In 'normal' operation conditions, 'distance', i.e. when two traders are 'close enough' to be influencing each other's actions, translates to ease of communication. Technology in its 'obsolete' form (telephone, telegraph, Telex, etc.) and, more recently, computerization and networking have made it possible for everybody to be in reach of everybody else. Physical distances no longer matter; traders do not hold a "fixed position" with respect to each other but are always in search of new correspondents, establishing new correlations. The energy that drives the elements of the stock market system to establish correlations is, in theory, economic growth and world development. Other factors, such as the availability of capital, trading information (or disinformation) etc., are equally important. Then, there are all the other multiply intricate social factors that social sciences try to decipher, in most cases to no avail. The stock market, as a mirror of human society, is incredibly more complicated than a fault network and even worse, it is subject to no known definitive theory; in short, the stock market is a social field that has no (physical) first principles and unknown fundamental equations. Interestingly enough however, it is possible to "... turn to the field of statistical physics for inspiration and guidelines" (Johansen and Sornette 1999).

Speculative bubbles and crashes are 'outliers' (Johansen and Sornette 1998), a term where in this case is used as a euphemism for abnormal situations. Such outliers are usually fuelled by excessive acquisitiveness, (in plain terms greed), which assumes the role of excessive energy supply and modulates mass psychology in a suggestive information environment. In this case, the 'investors' create among them a complex of short-range correlations by example and imitation: "My neighbour made money, so can I'. Long range correlations arise from the suggestive environment created by the general trend: "Other people make money, so can I". In the particular case of the 
1999 ASE bubble, a poll published in the newspaper "Kathimerini" (11 March 2001), shows that $53.48 \%$ of the sample has been driven to speculate as a result of a compulsive 'general clime', $25 \%$ because they 'admired' their friend's 'achievements', $9.9 \%$ for some 'other' cause, $7.8 \%$ at (compelling) familial suggestion and only $4.9 \%$ at the advise of stock brokers. In all, $78.8 \%$ of the 'investors' acted on blind faith and did not receive expert advice on how to trade their capital. On their part, traders mediate the interaction investors who speculate on the surplus value of stock investors who would like to speculate on the prospect of higher surplus. In this way, two antagonistic groups of traders develop: sellers and buyers. The interaction between these two groups depends on the capital offered by the investors and their cooperative response determines the value of the stock, which determines their (re)action and so forth... In this way, the stock market becomes a self-organizing system. The evolution of the market is modulated by fluidity (shifts) between the seller and buyer groups. As market price rises (buyers dominate) and prospects also rise, the shifts between sellers and buyers become more fluid and frequent. Eventually, there will be a point at which profiteering sellers will outnumber buyers. At this point a correction ensues, which will develop into a crash if the optimistic psychology that drove the buying rally reverses into panic driving a selling frenzy.

Recent research has presented evidence that speculative bubbles obey power laws with complex exponents, or equivalently discrete scale invariance: this translates to acceleration of the market price with log-periodic oscillations, i.e. oscillations periodic in the logarithm of the time-to-crash. The equation (Johansen and Sornette, 1999)

$$
F(t)=A+B \cdot\left(t_{c}-t\right)^{2} \cdot\left[1+C \cdot \cos \left(\omega \cdot \log \left(t_{c}-t\right)-\phi\right)\right]
$$

quantifies the time-to-crash of the bubble in terms of the price ending with a crash or large correction at a time close to $t_{c}$. This equation corresponds to a first order Fourier expansion of the general power law solution to a "renormalization equation"

$$
\frac{d F(t)}{d \log \left(t_{c}-t\right)}=n+i \omega
$$

around the "critical point" $t_{c}$. For all the bubbles in the most liquid markets, e.g., USA, Hong-Kong and the Foreign Exchange Market, the period $2 \pi / \omega$ has consistently been close to 1 (Johansen and Sornette 1999). Given that the period of the log-periodic oscillation corresponds to the preferred scaling ratio of the system (e.g. Sornette 1998), this value implies a scaling ratio approximately equal to $e \approx 2: 7$ : the local period of the log-periodic oscillations decreases according to a geometrical series with ratio $\approx e$. It has also been shown that although larger fluctuations were seen in the scaling ratio for a range of emergent markets, the statistics resulting from over twenty bubbles were quite consistent with that of the larger markets (Johansen and Sornette 2001).

\subsection{Data and results}

The data used in the present analysis is the General Index of the Athens Stock Exchange (ASE), which is a weighted sum of the stock value of a number of selected companies. The General Index (GI), as well as all other indices of the ASE, is available on-line from several sources; in this case the data was downloaded from the web pages of the financial newspaper "Naftemporiki" (at the URL http://www.naftemporiki.gr/markets). The identification of log-periodic power-law behaviour is rather straightforward: the GI time series is modelled with equation (7) using a Hedgehog non-linear optimisation procedure operating on the $\mathrm{L}_{2}$ norm. Raw data and model are shown in Figure 4.

It is apparent that after a period of significant variations during 1998, the ASE stock prices developed a phase of fast growth beginning on 20 October 1998. Putting aside the true reasons for the initial price increase, the growth soon evolved into trend-chasing that was inflated by unprecedented liquidity and unrealistic prospects, thus forming a bubble. The evolution of the GI shows clear signs of self-organization, exhibiting power-law acceleration with slow log-periodic oscilla- 
tion. Importantly enough, the frequency of the log-periodic oscillation is $\omega / 2 \pi \approx 0.37 \approx 1 / e$, consistent with the observations for other stock markets (Johansen and Sornette 1999). However, contrary to large liquid markets whose bubbles develop at slow rates and the log-periodic oscillation is apparent over an extended time-to-crash, the pace of growth in the ASE was very fast and lasted for only a few months. Perhaps this was due to the mass involvement of laypeople who introduced unprecedented liquidity, including earnings from illicit endeavours. The mass hysteria was so pervasive that even the 7 September earthquake did not impede the uncontrollable market growth; on the contrary, there have been reports of earthquake victims collectively speculating with their relief allowances and damage compensation (Kathimerini, 11 March 2001). The bubble burst at 20 September 1999 and was followed by a period of instability, downward trend and a spectacular crash during the next year. What is also important to note herein, is that the correction could be predicted with relative precision at any time after 1 August 1999!

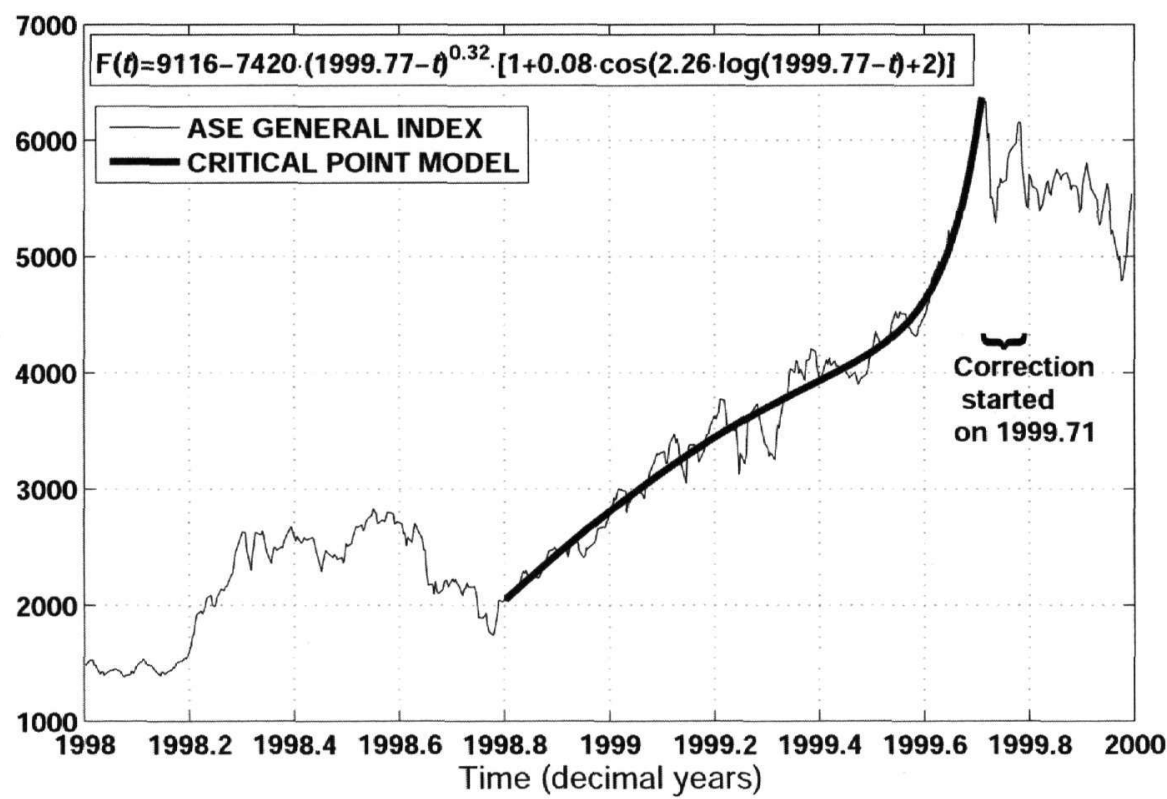

Figure 4 -The self-organizing process of the September 1999 ASE crisis. The thick black line corresponds to the model fitted with equation 7

\section{3. ...the ASE does it again!}

Apparently eager to emphasize its true nature, the ASE developed yet another phase of trendchasing during $2003-2006$, but this time much milder and with characteristics indicating a more mature market and economic environment (Fig. 5). This phase began after the ASE value hit a global low on the week ending 14 March 2003. The power-law acceleration of market growth exhibits definite log-periodic oscillation with frequency $\omega / 2 \pi \approx 1.48 \approx 4 / e$, almost quadruple of what is empirically expected - there's no apparent explanation for this observation. The process entered the critical phase on the week ending 3 March 2006, whereupon the market tried to hold its position during the next 5-6 weeks (meta-stable phase) but gave in with a correction by the week ending on 19 May 2006. To the Author's opinion, the definitive difference with the 1999 bubble is that this one was "professionally" driven: market growth was engineered by profiteering institutional investors rather than laypeople speculating en masse, which presumably rendered its 'mature market' characteristics (extended lifetime, apparent oscillatory behaviour, quieter burst and faster recovery). Details on this topic will be discussed elsewhere. 


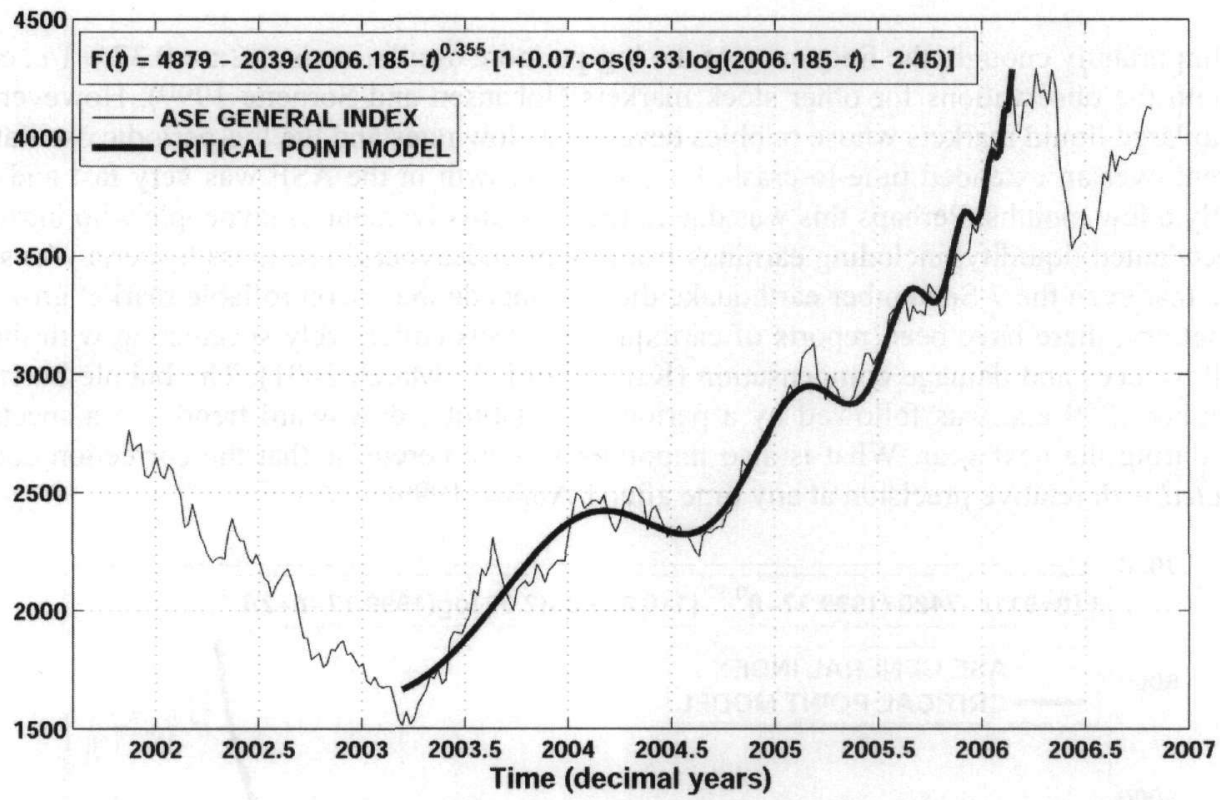

Figure 5 - The self-organizing process of the 2003-2006 ASE bubble. The observational data are weekly averages of the ASE General Index closing values

\section{Reflections}

This paper has briefly presented two cases of critical-point behaviour in very different selforganizing systems, coincidentally manifesting in Athens, Greece at September 1999. The first case pertains to the self-organization of the fault network centred on the fault that produced the M5.9 earthquake of 7 September, probably responding to remote excitation by the 17 August 1999, M7.4 Izmit earthquake. The earthquake generation process is empirically shown to obey continuous-scale invariance, (meaning that in can reproduce itself at any time or space scale), which is expressed in the form of pure power-law acceleration of seismic release rates. The second case pertains to the self-organisation of a considerably more complex system: a human network (society) and the processes/ interactions begetting the ASE bubble and stock-market crisis. The ASE growth process was different in that it obeyed discrete-scale invariance, meaning that it could only reproduce itself at quantized time or space scales; this is expressed in the form of power-law acceleration of market value decorated with log-periodic oscillations which modulate dynamic transitions between the discrete scales of the system. This type of behaviour appears to be typical of stock markets and reflects the fundamental geometry of the system's elements.

Now, consider that to the layman or to the novice, it would appear that earthquakes and stock markets have nothing in common. It might even appear ridiculous to hear one suggest that they do. The former are extensive and distributed systems of inanimate elements (fractures and cracks), quintessentially simple in their nature. The latter are complex systems of complex living beings: humans, self-aware and capable of constructive symbolic reasoning, who have used their abilities to master and reshape their world according to their requirements.

Or so the laypeople may think! As it turns out, when there are too many humans involved and too preoccupied with high expectations from a particular cause, reasoning and individuality "take a hike". The more the humans in a system and the higher their expectations, the less they are able to reason between them. Perhaps it is an oxymoron that reasoning will also allow recognition of this detail. But without reason, human masses become complex systems of simple animate elements they can undergo dynamic self-organisation and can, mutatis mutandis, behave like fractures and faults. 
Dynamic self-organizing complex systems are, so to speak, alive, albeit in a manner imperceptible to their individual elements: once formed, they grow and evolve. However, because their evolution is the resultant of the many-way (and frequently antagonistic) interactions between their elements, its macroscopic manifestation is, inevitably, not complex. In fact, it can be remarkably simple if one knows what to look for: power-law growth with, or without log-periodic oscillation. This appears to be universal and comprises the natural order for complex systems (also see Sornette, 1998, for additional information). So earthquakes and stock markets do have something in common after all: they both are subject to the same higher laws of the Natural Order. The same laws that appear to suggest another important thing: complex systems are not unpredictable!

One might contemplate whether the 'demotion' of the stock market, one of the pillars of societal endeavour, to a self-organizing system of simple animate elements, might mean something for the anthropocentrically oriented Human Society as a whole. Perhaps it suggests that the anthropic and analogous (religious in essence) principles, i.e. the basic tenets on which humans justify their view of themselves and the existence of the Universe, are "not even wrong" by Wolfgang Pauli's aphorism! Perhaps it suggests that Humanity is not as important to the 'eyes' of the general order of things, as humans like to think it is. However, these are questions without easy answers and a long future of discourse and debate!

\section{References}

Anderson, P., Arrow, K., and Pines, D., 1998. The Economy as a Complex Evolving System, Addison-Wesley.

Ben-Zion, Y., and Lyakhovsky, V., 2002. Accelerated seismic release and related aspects of seismicity patterns on earthquake faults, Pure appl. Geophys., 159, 2385-2412.

Bowman, D.D., and King, G.C.P., 2001. Accelerating seismicity and stress accumulation before large earthquakes, Geophys. Res. Letters, 38 (21), 4039-4042.

Bowman, D.D., Ouillon, G., Sammis, C.G., Sornette, A., and Sornette, D., 1998. An observational test of the critical earthquake concept, J. Geophys. Res., 103, 24359-24372.

Brodsky, E.E., Karakostas, V., and Kanamori, H., 2000. A new observation of dynamically triggered regional seismicity: Earthquakes in Greece following the August, 1999 Izmit, Turkey earthquake, Geophys. Res. Lett., 27, 2741-2744.

Bufe, C.G., and Varnes, D.J., 1993. Predictive modelling of the seismic cycle of the greater San Francisco Bay region, J. Geophys. Res., 98, 9871-9883.

Feigenbaum, J.A., and Freund, P.G.O., 1996. Discrete scale invariance in stock markets before crashes, Int. J. Mod. Phys., 10, 3737.

Gomberg, J., Beeler, N. M., Blanpied, M. L., and Bodin, P., 1998. Earthquake triggering by transient and stativ deformations, J. Geophys. Res., 103, 24411-24426.

Gomberg, J., Blanpied, M.L., and Beeler, N.M., 1997. Transient triggering of near and distant earthquakes, Bull. Seismol. Soc. Am., 87, 294-309.

Gutenberg, B., and Richter, C.F., 1956. The energy of earthquakes. Q. J. Geol. Soc. London, 112, $1-14$.

Hanks, T.C., 1977. Earthquake stress drops, ambient tectonic stresses and stresses that derive plate motions, Pure Appl. Geophys, 115, 441-458.

Harris, R.A., 1998. Introduction to special section: Stress triggers, stress shadows and implications for seismic hazard, J. Geophys. Res., 103, 24347-24358.

Herrmann, H.J., and Roux, S., eds., 1990. Statistical Models for the Fracture of Disordered Media, 353pp., Elsevier, Amsterdam. 
Huang, Y., Saleur, H., Sammis, C.G., and Sornette, D., 1998. Precursors, aftershocks, criticality and self-organized criticality, Europhys. Lett., 41, 43-48.

Johansen, A., and Sornette, D., 2001. Log-periodic power law bubbles in Latin-American and Asian markets and correlated antibubbles in Western stock markets: An empirical study, Int. J. Theo. Appl. Finance, 4 (6), 853. available from http://www.nbi.dk/ johansen/pub.html

Johansen, A., and Sornette, D., 2000. The NASDAQ crash of April 2000: Yet another example of log-periodicity in a speculative bubble ending in a crash, Eur. Phys. J. B, 17, 319-328.

Johansen, A., and Sornette, D., 1998. Stock market crashes are outliers, Eur. Phys. J. B, 1, 141143

Johansen, A., and Sornette, D., 1999. Modelling the stock market prior to large crashes, Eur. Phys. $J ., B, 9,167-174$.

“Kathimerini”, Sunday edition, 11 March 2001, pp 4 -6.

Keilis-Borok, V., 1990. The lithosphere of the Earth as a large nonlinear system, In G. D. Garland and J. R. Apel (eds), Quo Vademus: Geophysics for the Next Generation, Geophys. Monogr. Ser., AGU, Washington, D.C., vol. 60. 81-84,

Kertesz, J., and Kondor, I., 1998. Econophysics: An Emerging Science, Kluwer, Boston.

Rundle, J.B., Klein, W., Turcotte, D.L., and Malaud, B.D., 2000. Precursory seismic activation and critical point phenomena, Pure appl. Geophys., 157, 2165-2182.

Saleur, H., Sammis, C.G., and Sornette, D., 1996b. Discrete scale invariance, complex fractal dimensions, and log-periodic fluctuations in seismicity, J. Geophys. Res., 101, 17,661 - 17, 677.

Saleur, H., Sammis. C.G., and Sornctte, D., 1996a. Renormalization group theory of earthquakes, Nonlinear Processes in Geophysics, 3, 102-109.

Sammis, C.G., and Sornette, D., 2001. Positive feedback, memory and the predictability of earthquakes, e-print at http://arXiv.org/abs/cond-mat/0107143v1, July 2001.

Sornette, D., 1998. Discrete-scale invariance and complex dimensions, Phys. Rep., 297, 239.

Sornette, D., and Sammis, C.G., 1995. Complex critical exponents from renormalization group theory of earthquakes: Implications for earthquake predictions, J. Phys. 1, 5, 607-619.

Sornette. D., Vanneste, C., and Knopoff, L., 1992. Statistical model of earthquake foreshocks, Phys.Rev, A, 45, 8351-8357.

Tzanis, A., and Makropoulos, K., 2002. Did the 7/9/1999 M5.9 Athens earthquake come with a warning? Natural Hazards, 27, 85-103.

Tzanis, A., and Vallianatos, F., 2003. Distributed power-law changes and crustal deformation in the SW Hellenic Arc, Natural Hazards and Earth System Sciences, 3, 179-195.

Vandewalle, N., Ausloos, M. , Boveroux, P., and Minguet, A., 1998. How the financial crash of October 1997 could have been predicted, Eur. Phys. J. B, 4, 139-41.

Vanneste, C., and Sornette, D., 1992. Dynamics of rupture in thermal fuse models. J. Phys. I Fr. 2, 1621-1644.

Varnes, D.J., 1989. Predicting earthquakes by analysing accelerating precursory seismic activity, Pure appl. Geophys., 130, 661-686.

Voight, B., 1989. A relationship to describe rate-dependent material failure, Science, 243, 200203. 